\title{
Numerical and experimental investigation into the dynamics of a bubble-free-surface system
}

\author{
N. Bempedelis, ${ }^{1}$ J. Zhou, ${ }^{2}$ M. Andersson $\odot,{ }^{2}$ and Y. Ventikos ${ }^{1, *}$ \\ ${ }^{1}$ Department of Mechanical Engineering, University College London, London WC1E 7JE, United Kingdom \\ ${ }^{2}$ Department of Mechanics and Maritime Sciences, Chalmers University of Technology, \\ 41296 Gothenburg, Sweden
}

(Received 14 May 2020; accepted 8 January 2021; published 28 January 2021)

\begin{abstract}
The aim of the present work is to study the interaction between an oscillating bubble and a free surface. We perform a series of experiments and numerical simulations and attempt to characterize both early and late stages of the interaction. The focus is on providing insight into the mechanisms of bubble-induced atomization. For this reason, we are particularly interested in characterizing the patterns and dynamics of the liquid jets that are formed at the free surface. Observations regarding the evolution of the free surface are presented by measuring the jet's surface area and volume. Finally, based on these quantities, we introduce a metric that may be used to characterize the liquid jetting and predict whether late-time atomization of the interface will occur.
\end{abstract}

DOI: 10.1103/PhysRevFluids.6.013606

\section{INTRODUCTION}

Bubble dynamics has been an active topic of research for many years, owing to the regular presence of bubbles in our world and the large host of relevant applications, which range from medical treatment (e.g., shockwave lithotripsy $[1,2])$ to mechanical (e.g., cavitation-induced erosion [3]) and chemical [4] systems. Bubbles often grow or form near free surfaces. The most prominent example is the ocean, where bubbles bursting at the air-sea interface produce sea sprays, which strongly influence the coastal ecosystems and global climate patterns [5-9]. The dynamics of a bubble-free-surface system are also important to many technological applications, such as underwater explosions [10-12], printing or deposition techniques [13-15], drug delivery systems [16], and liquid fuel injection [17-21]. The bubbles in these applications may be oscillating, often quite violently. The interaction of the bubble with the free surface leads to the formation of liquid jets at the latter, an often desired outcome (e.g., aiding evaporation in liquid fuel injection).

The interaction between an oscillating bubble and a flat free surface was first studied theoretically and experimentally by Chahine [22] and Blake and Gibson [23]. The dynamics and patterns of the interaction were found to depend upon the ratio of the distance between the bubble and the surface to the maximum radius of the bubble. More recently, Patrascioiu et al. [24] performed a simultaneous visualization of both the bubble and free surface evolution, providing a better understanding of the correlation between bubble dynamics and liquid ejection. The formation of the initial spike jet and the subsequent thicker jet above the free surface were found to be related to the first expansion and rebound of the bubble, respectively. A parametric experimental study was performed by Zhang et al. [25], in an attempt to identify and categorize the different patterns that are observed during the bubble-surface interaction. The interaction of two collapsing bubbles beneath a free surface was studied by Ji et al. [26]. Both collapse and surface jets were found to be dependent on the interbubble

*y.ventikos@ucl.ac.uk 
distance and the bubble depth below the free surface. Kang and Cho [27] studied the evolution of the free surface following its interaction with a spark-generated bubble and proposed a relationship for the height of the surface jet when the interaction is weak (i.e., at large dimensionless bubble-surface distances, where the free surface deforms without breaking up).

Numerical investigations of the interaction were first carried out by Blake et al. [28] using the boundary integral method. Although simplified, the method was capable of describing the growth and collapse of a vapor bubble generated sufficiently far from the free surface. In general, the boundary integral method has been a popular choice for modeling the interaction of bubbles with surfaces [29-32], but is typically restricted to the early stages of the interaction. More recently, the problem was treated by Koukouvinis et al. [33] in an Eulerian framework, also taking into account liquid compressibility effects. The simulations were successful in predicting the bubble growth and collapse and in representing the fine details of the liquid-gas interface, such as the mushroom caplike structure at the tip of the jet and the splitting of the torus in an agglomeration of toroidal structures. The study focused on the dynamics of the bubble, and the motion of the free surface was only briefly mentioned. In a more recent work, Li et al. [34] studied the interaction between a gas bubble and a free surface using a similar numerical framework for a large range of dimensionless bubble-to-surface distances. The liquid jets at the free surface were characterized qualitatively, but discussion was as well focused on the characteristics of the bubble rather than the evolution of the free surface. Furthermore, both studies simulated only a small number of bubble oscillation cycles, reporting results up to the first stages of secondary jet formation.

In the present study, we characterize the evolution of the liquid jets at the free surface for timescales that extend beyond those of the oscillating bubble. The systems that are considered correspond to the "intermediate" regime [27], where the bubble oscillates without bursting at the surface, but the interaction is strong enough so as to induce atomization of the free surface at a later stage. As previously mentioned, past studies were mostly focusing on the dynamics of the bubble and were limited to its first few oscillation cycles. Furthermore, discussion revolved around quantities such as the jet height, width, or tip velocity that provide only limited insight into the evolution of the jet. Here we measure the surface area and enclosed volume of the free surface jet, providing further insight into its evolution. Based on these variables we are able to derive a phenomenological criterion that may be used to predict whether late-time atomization of the interface will occur. The paper is structured as follows: the experimental and numerical techniques that are used in the present study are detailed in the section that follows (Sec. II). Section III presents the set of experimental results, and Sec. IV presents the validation and results of the numerical simulations. Finally, results are analyzed and discussed (in relation to the process of bubble-induced atomization) in Sec. V.

\section{METHODOLOGY}

\section{A. Experimental setup}

The cavitation bubbles were generated by focusing laser light into deionized water at a wellcontrolled distance from the water-air interface. As shown in Fig. 1, the experimental setup consisted of two main parts: one for focusing laser into the cuvette to generate the cavitation bubble and another for performing shadowgraph imaging. In the laser focusing part, a Nd:YAG laser (SpectraPhysics LAB170/10) generated $6 \mathrm{~ns}$-duration laser pulses with a $532 \mathrm{~nm}$ wavelength. The laser pulses were attenuated by an attenuator composed of a half wave plate and a Glan laser prism. The energy of the laser pulse was adjusted by rotating the half wave plate, which was installed in a precision rotation mount. The laser beam was expanded by the concave lens L1 $(-20 \mathrm{~mm})$ and the focal lens L2 $(+100 \mathrm{~mm})$. The expanded beam was focused into a glass cuvette in the shape of a $50 \mathrm{~mm}$ cube by the focal lens L3 $(+75 \mathrm{~mm})$. The cuvette was filled with deionized water, and the laser light was focused beneath the water surface. The laser focus was fixed while the height of the cuvette could be adjusted. In this way, the distance between the laser-induced cavitation bubble and 


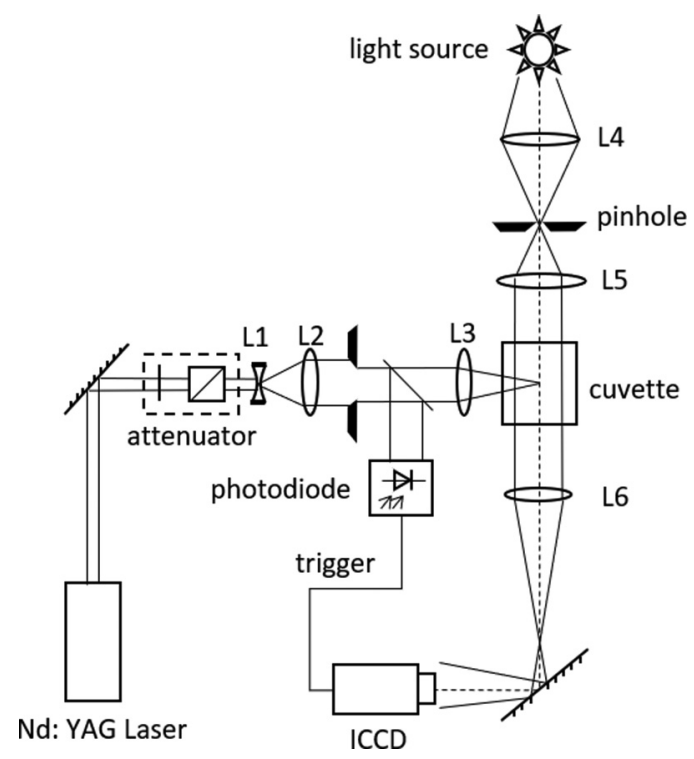

FIG. 1. Schematic of the experimental setup.

the free surface could be varied. In the shadowgraph part, a plasma lamp (THORLABS HPLS200) was used as the light source. The light was focused by a microscope L4 with 20 times magnification and a numerical aperture of 0.4 . The focused light passed through a pinhole whose diameter was $300 \mu \mathrm{m}$ and was collimated by the focal lens L5. The collimated light passed through the cuvette and was focused by the focal lens L6. A shape edge could be put at the focus point after L6 to perform Schlieren imaging with the same setup. An intensified CCD (ICCD) camera (Princeton Instruments, PI-Max 4) with a $180 \mathrm{~mm}$ lens (Nikon) and $2 \times$ teleplus (Kenko) was used to capture the images. The camera was triggered by a photodiode which detected the laser light reflected by a window inserted between L2 and L3. The exposure time was typically $3 \mathrm{~ns}$.

\section{B. Numerical simulations and computational setup}

The flow was modeled by the compressible Euler equations, together with an equation for the total energy. The effects of surface tension and gravity were also included, as they are essential for the accurate description of the free surface dynamics [27]. The fluids were assumed immiscible, while the effects of diffusion (thermal and viscous) and phase change were neglected. The former may be justified by consideration of the relevant dimensionless numbers and of the relative magnitude of diffusivity coefficients; the Ohnesorge number can be estimated to be of the order of $O\left(10^{-3}\right)$ (based on the maximum bubble radius and the liquid properties), suggesting that viscous forces are small compared to inertial and surface tension ones. This argument can be extended to all diffusion processes. Phase change was present in the experiments, but is of secondary importance in the numerical simulations, due to the approach that was adopted for the modeling of the bubble and its contents. Similar assumptions are made in most relevant studies [23,28-34] and have led to overall successful predictions. This is observed in the present work as well, as the simulations are shown to be in agreement with the experimental results. The system of equations was closed with the stiffened gas equation of state, which relates the pressure with the density and internal energy of the fluid,

$$
p+(\Gamma+1) p_{\infty}=\rho \Gamma\left(e+e_{\infty}\right)
$$


TABLE I. Thermodynamic parameters for the stiffened gas equation of state.

\begin{tabular}{lccc}
\hline \hline Fluid & $\Gamma$ & $p_{\infty}(\mathrm{Pa})$ & $e_{\infty}(\mathrm{J} / \mathrm{kg})$ \\
\hline Water & 3.4 & $6 \times 10^{8}$ & $7.456 \times 10^{6}$ \\
Air & 0.4 & 0 & 0 \\
\hline \hline
\end{tabular}

where $\Gamma$ is the Grüneisen exponent, $p_{\infty}$ the stiffened pressure constant, $e$ the specific internal energy and $e_{\infty}$ the energy translation factor. For the fluids considered in this study, these values are taken from Hawker and Ventikos [35] and are given in Table I.

Simulations were performed using a well-established high-fidelity front tracking framework [36-40] coupled with the grid-aligned ghost fluid method [41,42], enabling the fully sharp representation of the interfaces and the jumps of variables across them. A fifth-order WENO scheme was used for the calculation of the fluxes, and a second-order Runge-Kutta method for time integration. This framework has been validated and used for bubble problems in previous works by the authors [41,43]; the interested reader is referred to Refs. [41,42] for more details on the employed numerical method.

In order to accurately replicate the experimental conditions, accounting for the presence of the cuvette was desired. This raised the computational cost considerably, as the size of the cuvette $(50 \mathrm{~mm} \times 50 \mathrm{~mm} \times 50 \mathrm{~mm})$ was much larger compared to the maximum bubble radius $(\simeq 1 \mathrm{~mm})$. As a result, performing full three-dimensional simulations was not feasible. Instead, the problem was modeled by the axisymmetric form of the flow governing equations. At this point a minor discrepancy exists, as the cubic cuvette was modeled as a cylindrical container. However, this is not expected to have a significant effect on the flow, as the cuvette walls are sufficiently far (based on a number of test simulations with varying cuvette sizes). The size of the computational domain was therefore equal to $x \in[0 \mathrm{~m}, 0.025 \mathrm{~m}], y \in[-0.05 \mathrm{~m}, 0.0125 \mathrm{~m}]$. Reflecting boundary conditions were used to model the side and bottom cuvette walls (at $x=0.025 \mathrm{~m}$ and $y=-0.05 \mathrm{~m}$, respectively) and symmetry conditions were applied along the axis of symmetry $(x=0 \mathrm{~m})$. Nonreflecting conditions were applied at the remaining boundary. For all cases considered in this work, the free surface was placed at $y=0 \mathrm{~m}$, and the bubble was placed at varying distances below it.

The laser-generated bubble was modeled as a high-pressure air bubble, as in Refs. [33,34]. However, the initial conditions of the bubble (size, pressure, and temperature) were unknown. These were adjusted so as to match the size and period of the bubble for one of the experimental cases. Still, there exist a large number of conditions that yield results in accordance with the experiment. For reasons related to computational cost, the initial bubble cannot be infinitely small. On the other hand, if the bubble has already expanded significantly, one would have to introduce the associated velocity field. This was avoided by considering bubbles of sufficiently small initial size.

Definition of the initial bubble was achieved by scanning the phase space of conditions (size, pressure, and temperature). In an attempt to narrow down the parametric domain, the bubble was assumed to be in thermal equilibrium with its surroundings. Bubbles were eventually introduced with an initial radius of $R_{\mathrm{in}}=0.00015 \mathrm{~m}$, about an order of magnitude smaller than their maximum size (bubbles were reported to grow $\simeq 100$ times the size of the original plasma [44]). Such a value was found to be sufficient for a proper description of the bubble growth, while keeping the required resolution to realizable levels. An initial pressure of $p_{\text {in }}=201 \mathrm{~atm}$, corresponding to an initial bubble energy of $E_{\mathrm{pot}}=\Delta p\left(4 \pi R_{\mathrm{in}}^{3}\right) / 3=0.29 \mathrm{~mJ}$, about $5 \%$ of the laser pulse energy, was found to give results in accordance with the experiment.

Other than the bubble interior, both fluids were initialized at atmospheric conditions. The domain was discretized with a resolution corresponding to 12 points per initial bubble radius, yielding a total number of $10^{7}$ elements. As shown in Ref. [41], such a resolution is sufficient for the prediction of both growth and collapse stages. This will be further verified in Sec. IV, where the numerical results are compared with the experimental ones. 
TABLE II. Conditions and parameters of experimental configurations.

\begin{tabular}{lccc}
\hline \hline Case & $\delta(\mathrm{mm})$ & $R_{\max }(\mathrm{mm})$ & $\gamma$ \\
\hline E-0.86 & 1.12 & 1.29 & 0.86 \\
E-0.94 & 1.02 & 1.08 & 0.94 \\
E-1.10 & 1.36 & 1.24 & 1.10 \\
E-1.27 & 1.60 & 1.26 & 1.27 \\
\hline \hline
\end{tabular}

\section{EXPERIMENTAL RESULTS}

In this section, an overall description of the bubble dynamics and induced surface deformations is presented, based on the shadow images. More detailed data determined from the experimental images will be presented in the sections that follow, along with the numerical results.

The dimensionless parameter $\gamma=\delta / R_{\max }$ (also referred to as nondimensional stand-off parameter), where $\delta$ is the distance between the bubble center and the free surface and $R_{\max }$ is the maximum bubble radius, was considered as a measure of comparison between the different experimental conditions [24]. The laser pulse energy was adjusted to $\simeq 6 \mathrm{~mJ}$, so the maximum bubble radius was almost the same for each case. Different $\gamma$ values were achieved by varying the distance $\delta$. A total of four cases were considered in this study. The values describing each configuration are presented in Table II.

Examples of shadowgrams of the collapsing bubbles and free surface deformations in different conditions are shown in Fig. 2. Results from case E-0.94 will be presented in a following section, together with the results of the numerical method. The time-resolved images were obtained by capturing images at different time delays from the laser pulse. The bubble and surface deformation events were highly reproducible, thus it was straightforward to construct a sequence from images recorded at different bubble-induced events. The presented images are representative examples from a set of at least five images recorded at the same time step and conditions.

The horizontal black line slightly below the middle of the images shows the position of the water-air interface. Below it, shadows of the bubble can be seen. At early times, during the first growth period, the bubble retained an almost spherical shape and reached its maximum size around $t=0.1 \mathrm{~ms}$. The bubble then assumed a nonspherical shape while collapsing (before or around $t=0.2 \mathrm{~ms}$ ). The top of it, i.e., the side close to the free surface, was pushed downwards into the bubble by a liquid jet [45]. This is the reason why the upper surface of the bubbles appears flat in the images at $t=0.15 \mathrm{~ms}$ in Figs. 2(a) and 2(b). Torn by the downwards liquid jet, the top of the bubble adopted the shape of a torus as it was shrinking, looking like a thick line segment in the side view as seen in the images at $t=0.2 \mathrm{~ms}$ in Figs. 2(a) and 2(b). During later stages, such as the rebound and recollapse, the bubble separated into multiple parts and moved away from the surface, shown at $t=0.5 \mathrm{~ms}$ in Figs. 2(a) and 2(b) and at $t=0.4 \mathrm{~ms}$ in Fig. 2(c).

Above the interface, the evolution of the surface deformations, jets, and protrusions can be followed. In general, the bubble-induced free surface deformations can be divided in two stages. The first one is the spikelike jet formed before the rebound of the bubble, as shown in the images at $t=0.1 \mathrm{~ms}$ and $t=0.15 \mathrm{~ms}$ in Figs. 2(a) and 2(b). This spike jet forms a counterjet pair with the one penetrating the bubble from the top as described above [46]. The second stage is the thick jet formed after the first collapse and rebound of the bubble, as shown in the images after $t=0.3 \mathrm{~ms}$ in Fig. 2(a), the images after $t=0.5 \mathrm{~ms}$ in Fig. 2(b), and the images after $t=0.6 \mathrm{~ms}$ in Fig. 2(c).

In the case where $\gamma=0.86$, the spike jet was faster than the thick jet. In the case where $\gamma=$ 1.10 , although the spike jet started earlier, the thick jet grew faster and engulfed the spike jet after $t=1.5 \mathrm{~ms}$. The engulfed jet was also observed by Zhang et al. [25]. For the case where $\gamma=1.27$, 
(a)

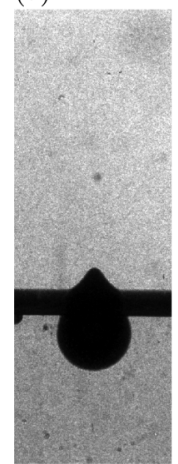

$0.10 \mathrm{~ms}$

(b)

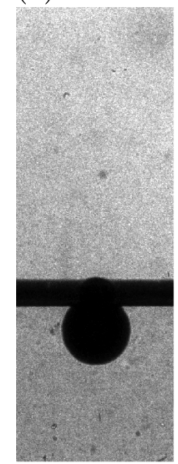

$0.10 \mathrm{~ms}$

(c)

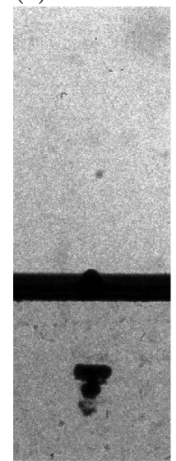

$0.40 \mathrm{~ms}$

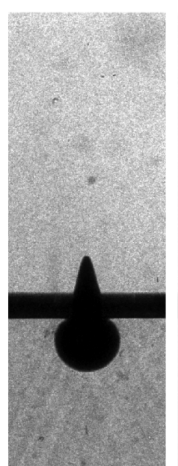

$0.15 \mathrm{~ms}$

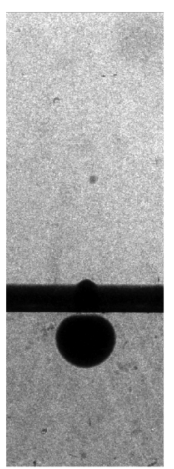

$0.15 \mathrm{~ms}$

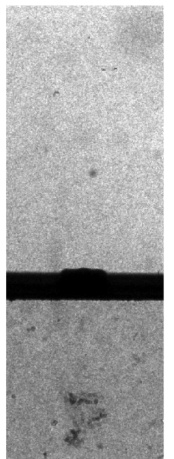

$0.60 \mathrm{~ms}$

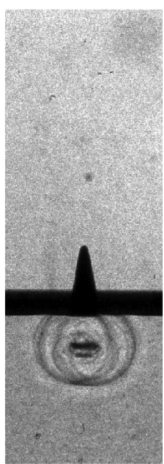

$0.20 \mathrm{~ms}$

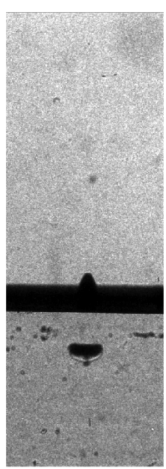

$0.20 \mathrm{~ms}$

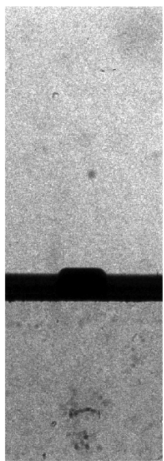

$0.70 \mathrm{~ms}$

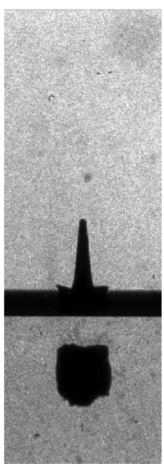

$0.30 \mathrm{~ms}$

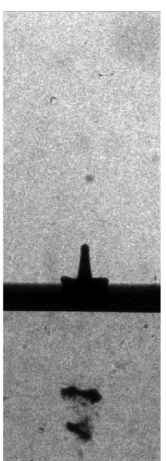

$0.50 \mathrm{~ms}$

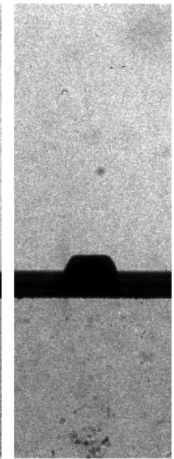

$1.00 \mathrm{~ms}$

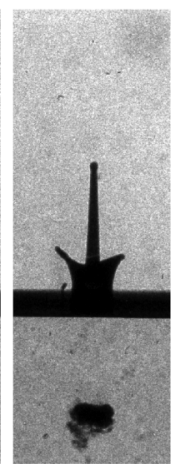

$0.50 \mathrm{~ms}$

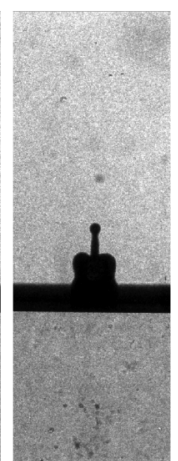

$1.00 \mathrm{~ms}$

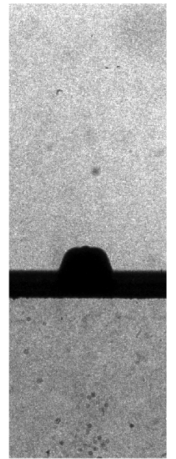

$1.30 \mathrm{~ms}$

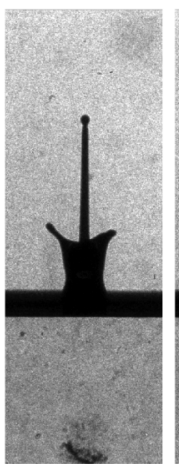

$0.70 \mathrm{~ms}$

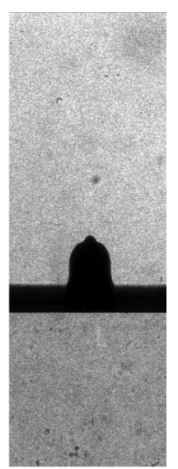

$1.50 \mathrm{~ms}$

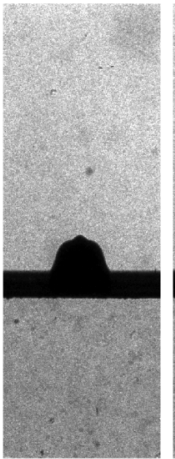

$1.50 \mathrm{~ms}$

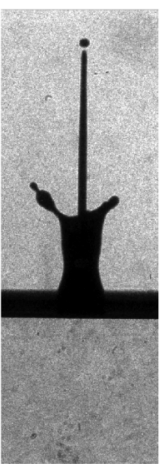

$0.90 \mathrm{~ms}$

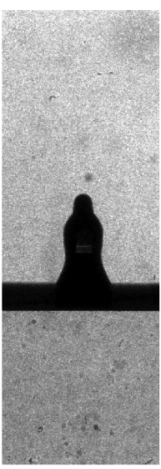

$2.00 \mathrm{~ms}$

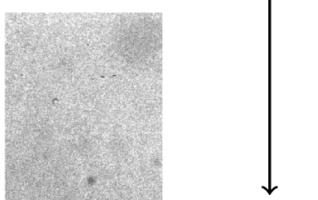

$2.00 \mathrm{~ms}$

FIG. 2. Shadowgrams of the collapsing bubbles and free surface deformations at different conditions. (a) Case E-0.86, (b) case E-1.10, (c) case E-1.27. Depicted window size $6 \mathrm{~mm} \times 17 \mathrm{~mm}$.

there was no spike jet but a small deformation on the surface, as shown in the image at $t=0.4 \mathrm{~ms}$ in Fig. 2(c). At $t=0.6 \mathrm{~ms}$, both the tip of the small deformation and a wider deformation around the tip in the center were observed. After $t=0.7 \mathrm{~ms}$ this small deformation was no longer visible; the thick jet was instead rising with an initially flat top, which later developed a peak in the center. The shapes of the thick jet front were different in these cases. For $\gamma=0.86$, the thick jet front was more violent with a crown-like shape (thus also referred to as a crown jet), while in the case of $\gamma=1.10$ it was more rounded. When $\gamma=1.27$, the thick jet had initially a flat-shaped front. The cylindrical shape of the thick jet is induced by the toroidal geometry of the rebounding bubble [24]. 
TABLE III. Conditions and parameters of numerical configurations.

\begin{tabular}{lccc}
\hline \hline Case & $\delta(\mathrm{mm})$ & $R_{\max }(\mathrm{mm})$ & $\gamma$ \\
\hline C-0.61 & 0.650 & 1.072 & 0.61 \\
C-0.77 & 0.840 & 1.097 & 0.77 \\
C-0.92 & 1.017 & 1.105 & 0.92 \\
C-1.06 & 1.180 & 1.110 & 1.06 \\
C-1.22 & 1.361 & 1.116 & 1.22 \\
\hline \hline
\end{tabular}

\section{NUMERICAL RESULTS}

\section{A. Validation}

In the present section, we demonstrate the validity of our simulations by performing a comparison with the experimental results (for the conditions denoted as case E-0.94 in Table II). A qualitative comparison between the numerical (denoted as C-0.92; see Table III) and experimental results at different time instants is presented in Fig. 3. Excellent agreement is observed for the evolution of both the bubble and the free surface. As the bubble expands $(t=0.10 \mathrm{~ms})$, the free surface is

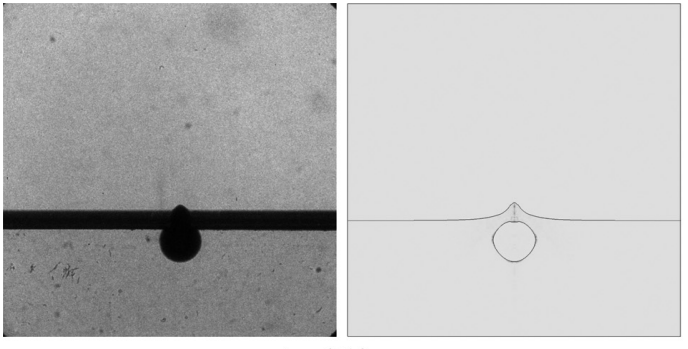

$$
t=0.10 \mathrm{~ms}
$$

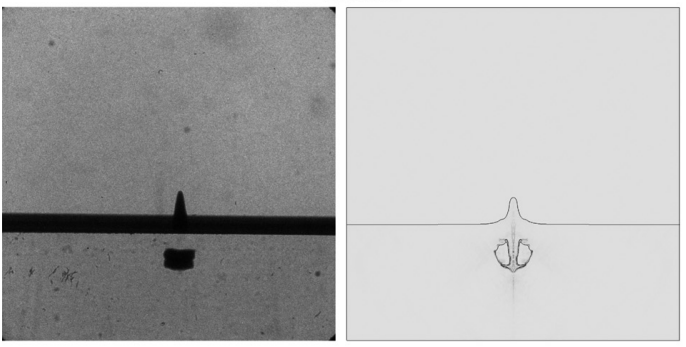

$$
t=0.20 \mathrm{~ms}
$$

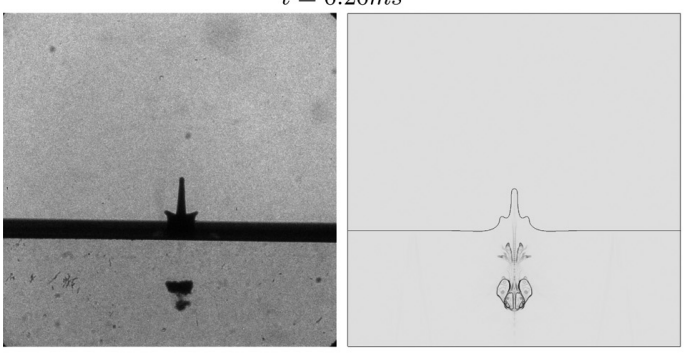

$t=0.40 \mathrm{~ms}$

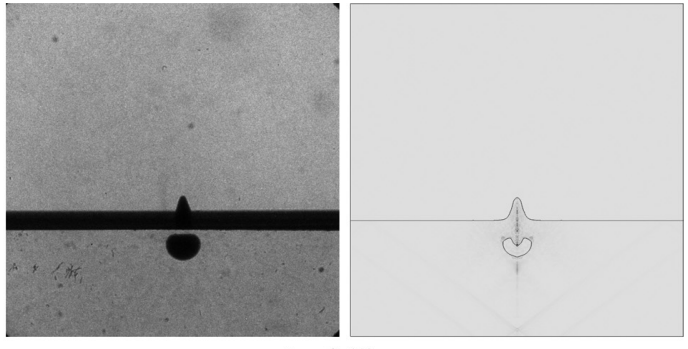

$t=0.15 \mathrm{~ms}$

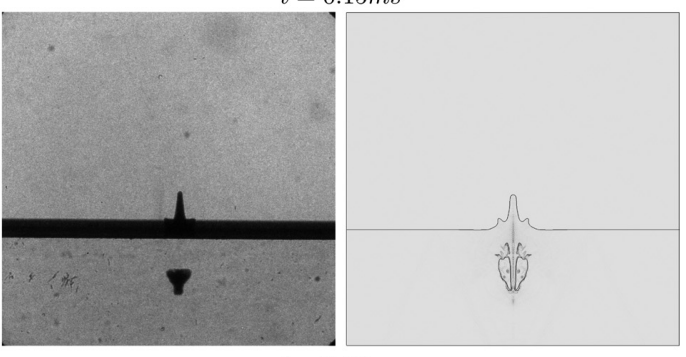

$t=0.30 \mathrm{~ms}$

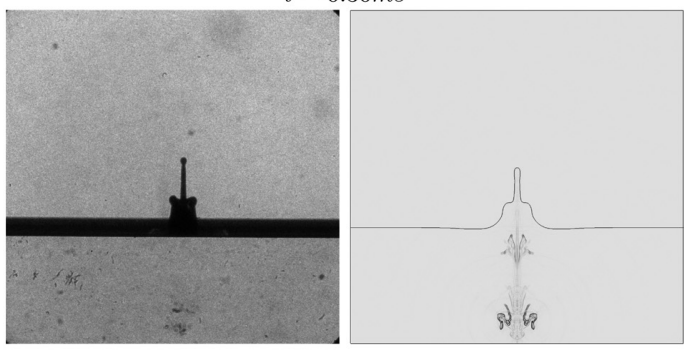

$t=0.70 \mathrm{~ms}$

FIG. 3. Comparison between (left) experimental (case E-0.94) images and (right) numerical (case C-0.92) results at different time instants following the generation of the bubble. Computational results are visualized via numerical Schlieren (magnitude of density gradient) contours. Depicted window size $17 \mathrm{~mm} \times 17 \mathrm{~mm}$. 
(a)

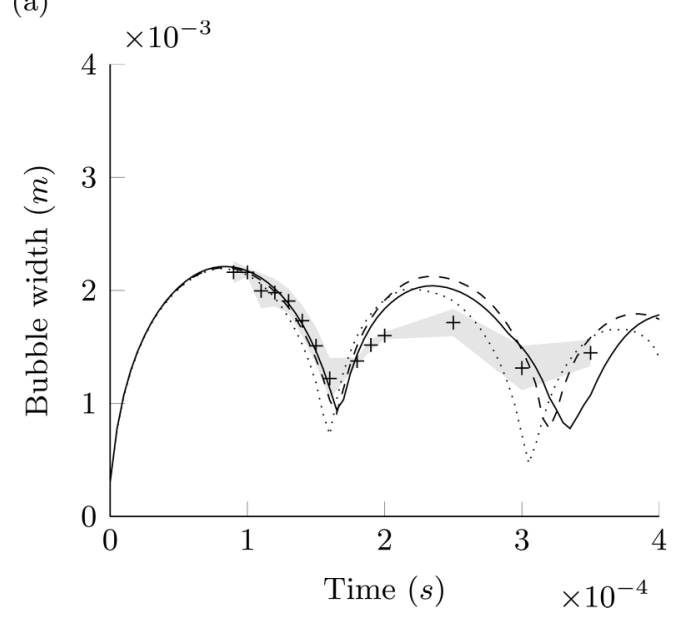

(b)

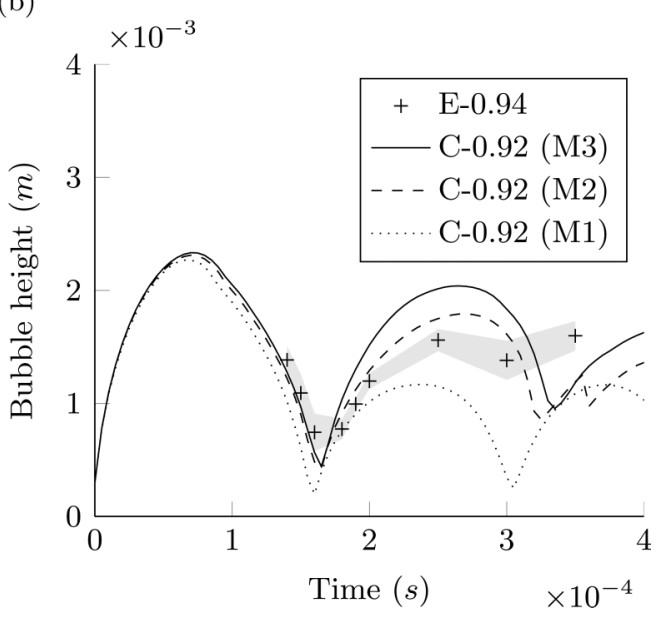

FIG. 4. Time history of bubble evolution. (a) Bubble width, (b) bubble height. The shaded region around the markers represents experimental uncertainty.

deformed, leading to the formation of the main (spike) liquid jet. The counterjet that is subsequently formed $(t=0.15 \mathrm{~ms}$ ), pierces the bottom bubble wall, which then assumes a toroidal shape $(t=0.20 \mathrm{~ms})$. The bubble rebounds and then collapses for a second time, around $t=0.335 \mathrm{~ms}$. During this cycle, the crown-shaped thick jet is formed. In the cycles that follow, the bubble assumes various complex shapes while radiating energy at every collapse. During these cycles, the tip of the crown jet recedes (more evident in the absence of asymmetries and interfacial instabilities).

A quantification of the results is presented in Figs. 4 and 5. Results from two additional simulations with discretizations corresponding to nine and six points per initial bubble radius (resulting in meshes of $5.625 \times 10^{6}$ and $2.5 \times 10^{6}$ elements, denoted as M2 and M1, respectively) are presented along with the results of the mesh used throughout the study (described in Sec. II, labeled M3). In Fig. 4 the time histories of the bubble width and height are plotted for the first two and a half bubble cycles, where experimental data are available for comparison. The dynamics of the first cycle

(a)

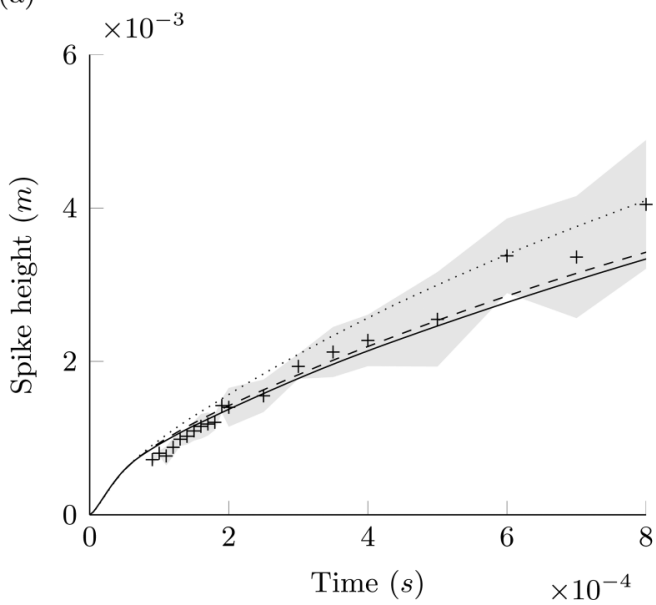

(b)

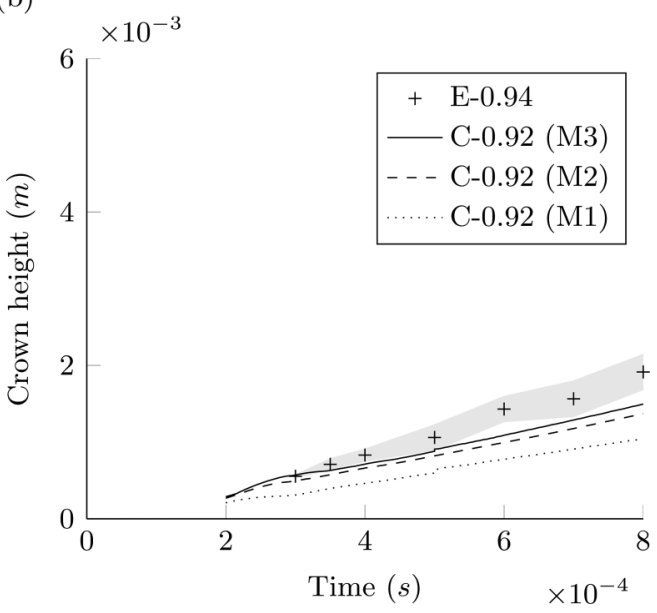

FIG. 5. Time history of free surface deformation. (a) Spike height, (b) crown height. The shaded region around the markers represents experimental uncertainty. 
are in excellent agreement with the experimental results for all grid resolutions. However, numerical simulations predict a larger rebound for the bubble. This is partially attributed to the modeling of the bubble contents; the elastic behavior of the gas results in larger rebounds (i.e., a bigger percentage of energy is stored in the bubble than being radiated away via shock waves) [47]. As the bubble has more energy, the second collapse is slightly delayed. However, we note that there is another reason for the overestimation of the bubble height: numerical results (except those of mesh M1, which fails to predict the split of the bubble in toroidal structures) include the very thin (subgrid) filaments that were detached from the bubble following the impact of the liquid jet at the bottom bubble wall [e.g., Fig. 3(c)].

With respect to the evolution of the free surface, the spike- and crown-like jets that were observed in the experiments were also predicted by the simulations (see Fig. 3). In Fig. 5 the temporal evolution of the spike and crown jet heights is plotted until the bubble has traveled sufficiently far away and radiated all of its energy away. Up to this moment $(t=0.8 \mathrm{~ms})$, the bubble has completed seven cycles. Moreover, this is the moment when instabilities start taking over the atomization process in the experiment, with the formation of drops at the tip of the jet. The idealized conditions of the simulations in comparison to the experiments (e.g., perfectly spherical initial bubble and flat free surface) delay somewhat the development of such instabilities. Overall, the trend of jet evolution is accurately predicted by the simulations with grids M2 and M3. However, the height of both jets at late-time stages is slightly underestimated.

\section{B. Parametric study}

Following the validation of the numerical method against the experimental results, a series of simulations was performed in an attempt to characterize the process of bubble-induced atomization. Four additional cases (with a computational setup similar to the one described in Sec. II) were simulated, with the only parameter changing being the distance between the bubble and the free surface. The conditions of the numerical configurations are presented in Table III. The data presented in this section are extracted from the simulations every $t=5 \mu \mathrm{s}$.

A visual overview of the evolution of the bubble-free-surface system for the different nondimensional stand-off parameters $\gamma$ is presented in Fig. 6. The process is depicted by plotting the pressure field along with the liquid-gas interfaces at four different time instants for each case. It is clear that the interaction is stronger when the bubble is placed closer to the free surface. This is reflected not only in the free surface jet, but also in the decrease of the expansion of the bubble in the horizontal direction (see Table III), the decrease of the bubble collapse time, and the stronger and thinner bubble microjets (in agreement with other works in the literature, e.g., Ref. [34]). During early stages, shortly after the second bubble cycle (around $t=0.3 \mathrm{~ms}$ ), the free surface has formed both spike and thick jets for all nondimensional stand-off parameters under consideration (in the case where $\gamma=1.22$ this refers to the three distinct tips at $t=0.325 \mathrm{~ms}$ ). At later stages, the tips of the thick jets become less pronounced or get entirely engulfed, while for large $\gamma$ values the thick jets merge with the main one, forming a single jet. It is observed that for the cases where $\gamma=0.61$, $\gamma=0.77$, and $\gamma=0.92$ the interface of the spike jet displays Rayleigh-Plateau instabilities, which lead to the formation and detachment of drops at a later stage. Overall, the results compare well with the corresponding experimental cases (Fig. 2); the predicted interaction patterns are in good agreement.

\section{DISCUSSION}

Other than presenting the different bubble-free-surface interaction patterns, the aim of the study is to characterize the potential for atomization induced by the oscillation of bubbles. In that respect, numerical simulations provide easy access to a plethora of useful data. In Figs. 7(a) and 7(b) the deformation of the free surface is quantified by tracking its maximum height and its width close to the root (at $0.7 \mathrm{~mm}$ above its initial position). Results are nondimensionalized using the maximum 

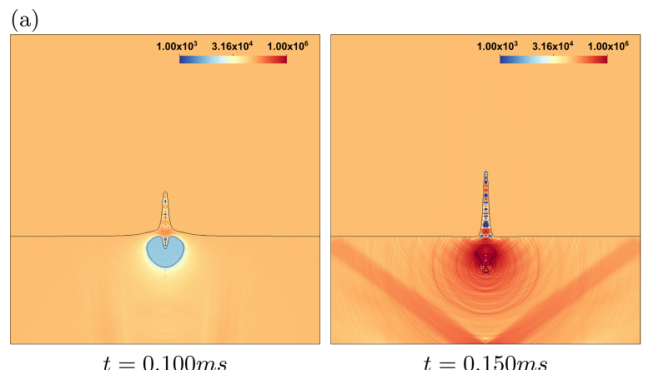

$t=0.150 \mathrm{~ms}$
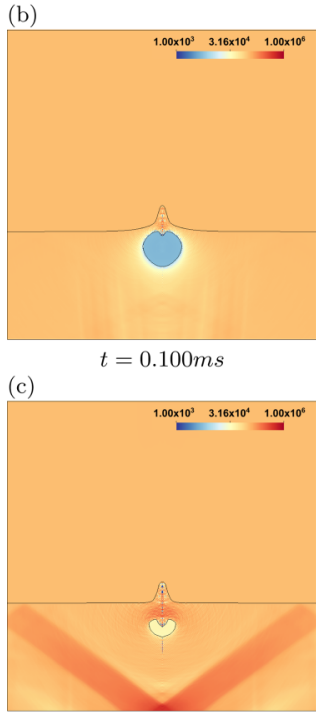

$t=0.150 \mathrm{~ms}$

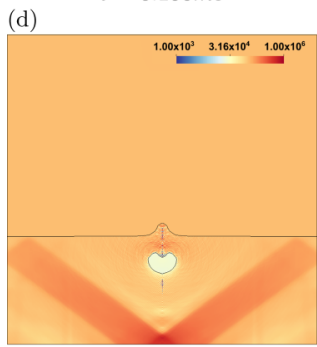

(e)

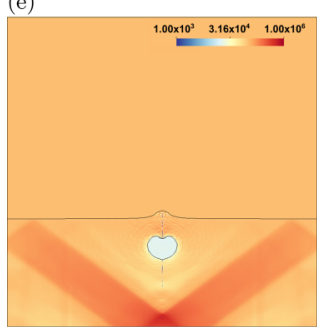

$t=0.150 \mathrm{~ms}$

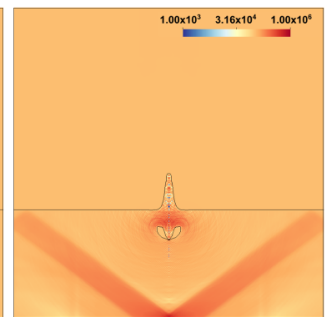

$t=0.150 \mathrm{~ms}$

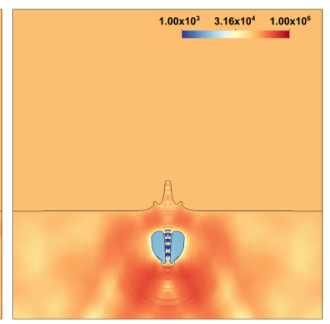

$t=0.275 \mathrm{~ms}$
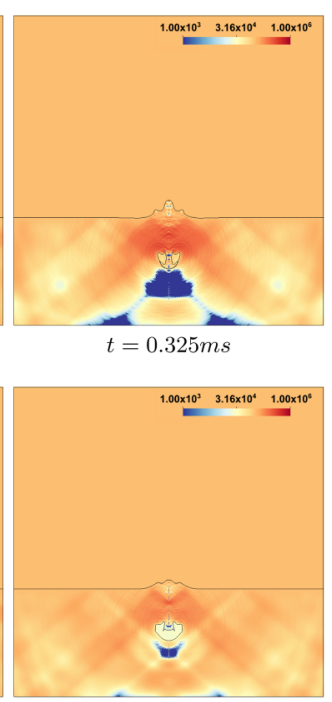

$t=0.325 \mathrm{~ms}$

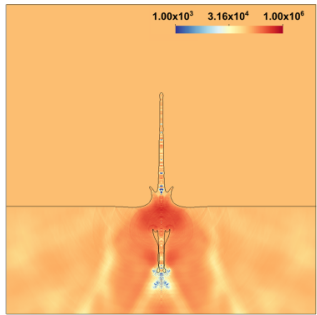

$t=0.275 \mathrm{~ms}$

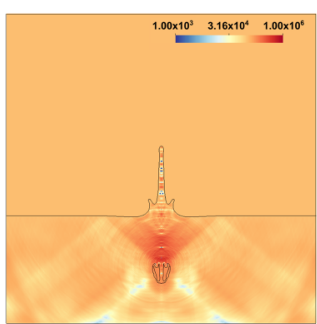

$t=0.325 \mathrm{~ms}$

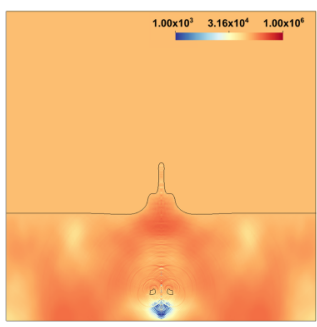

$t=0.600 \mathrm{~ms}$

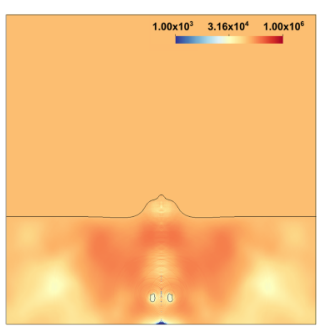

$t=0.700 \mathrm{~ms}$

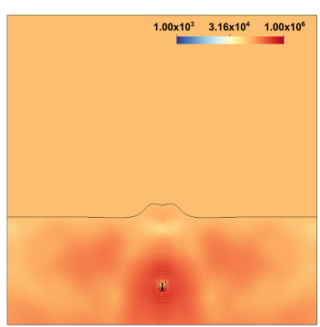

$t=0.800 \mathrm{~ms}$

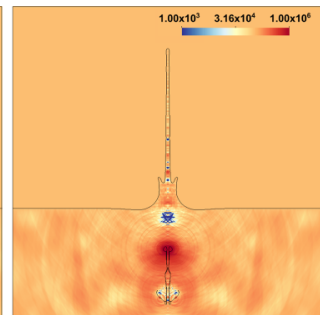

$t=0.400 \mathrm{~ms}$

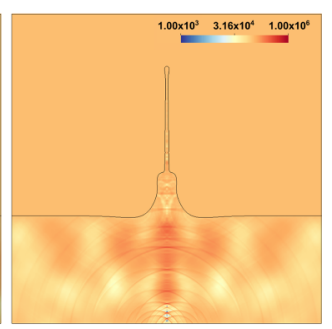

$t=0.800 \mathrm{~ms}$

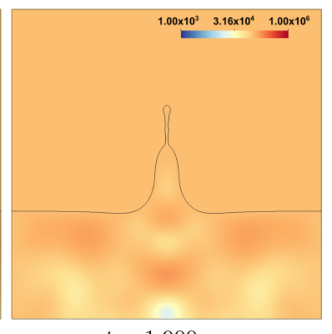

$t=1.900 \mathrm{~ms}$

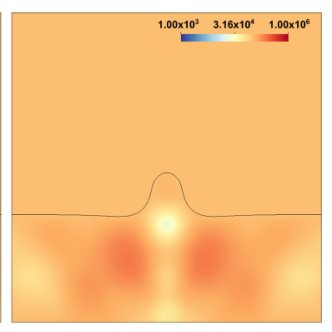

$t=1.900 \mathrm{~ms}$

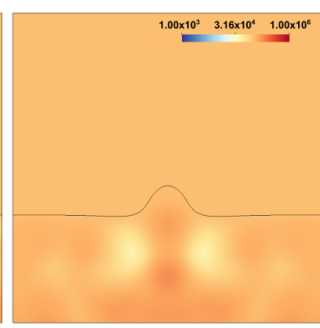

$t=1.900 \mathrm{~ms}$

FIG. 6. Pressure (Pa) contours along with gas-liquid interfaces for different nondimensional stand-off parameters. (a) Case C-0.61, (b) case C-0.77, (c) case C-0.92, (d) case C-1.06, (e) case C-1.22. Depicted window size $17 \mathrm{~mm} \times 17 \mathrm{~mm}$. 
(a)

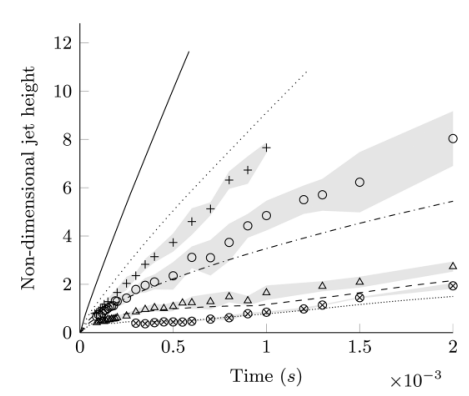

(b)

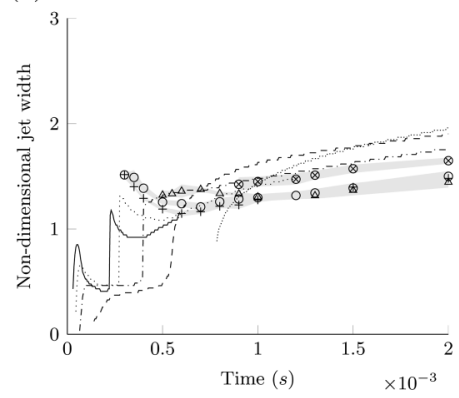

(c)

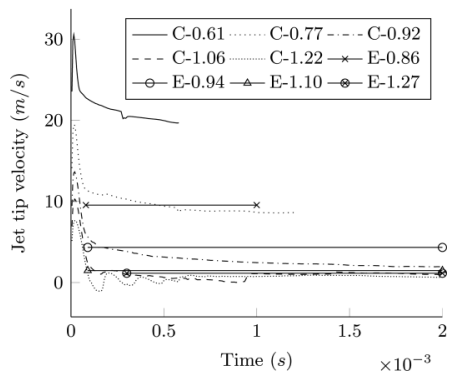

FIG. 7. Time history of jet evolution for the considered configurations. (a) Nondimensional jet height, (b) nondimensional jet width. The shaded region around the markers represents experimental uncertainty. (c) Jet tip velocity. Markers denote the beginning and end of the experimental data averaging period.

bubble radius (presented in Tables II and III). Numerical predictions are plotted along with the experimental results, showing similar trends for the evolution of the free surface jet as a function of the nondimensional stand-off parameter $\gamma$. Complementing the visual observations from Fig. 6, it is found that the jets become longer and faster as the bubble gets closer to the free surface while maintaining a rather constant width close to their root. The time history of the velocity of the jet tip is presented in Fig. 7(c). Again, numerical results are compared with experimental ones (which are averaged over the time period denoted by the start and end markers). At the initial stages corresponding to the first growth cycle of the bubble, the free surface deforms very rapidly. At later stages, jet tip velocity asymptotically progresses towards a constant value, different for each nondimensional stand-off parameter.

Figure 8(a) shows the generated surface area for the initially flat free surface. Three distinct phases (labeled in the figure as I, II, and III) of surface area generation may be distinguished (more easily for the cases where $\gamma=0.61, \gamma=0.77$, and $\gamma=0.92$ ), corresponding to the first, second, and later bubble cycles, respectively. The largest slope, i.e., where surface area is most rapidly generated, is observed for the second bubble cycle, which is the cycle where the thick jet is formed. Broadly speaking, surface area generation can be assumed to be a linear function of time, particularly for larger $\gamma$ values. Figure 8(b) depicts the volume of the free surface jet (volume of liquid above the $y=0$ line). The nonmonotonous behavior of the volume against time is a result of the growing bubble creating a wide, but not very high, bump, as can be seen in the images at $t=0.1 \mathrm{~ms}$ in Figs. 2 and 3. This short-lived bump does not contribute much to the change in surface area, but due to its relatively large width, the volume above the original surface level is large

(a)

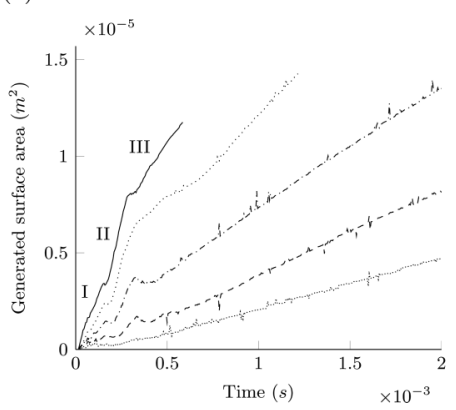

(b)

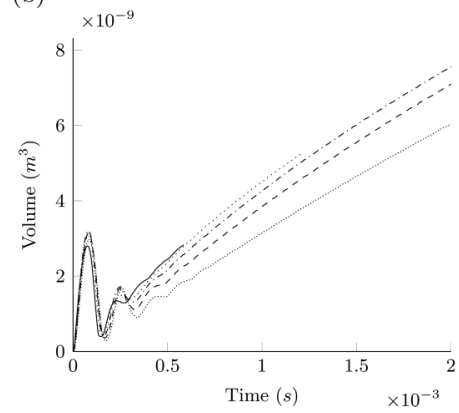

(c)

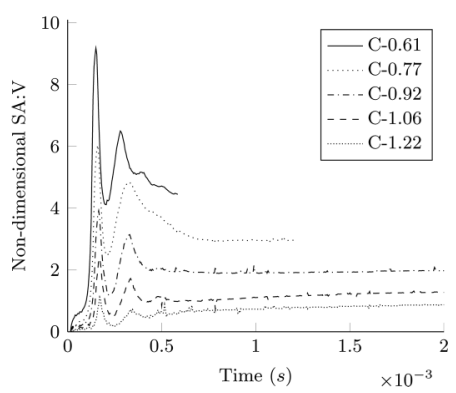

FIG. 8. Time history of jet evolution for different nondimensional stand-off parameters. (a) Generated surface area, (b) volume, and (c) nondimensional surface-area-to-volume ratio. 


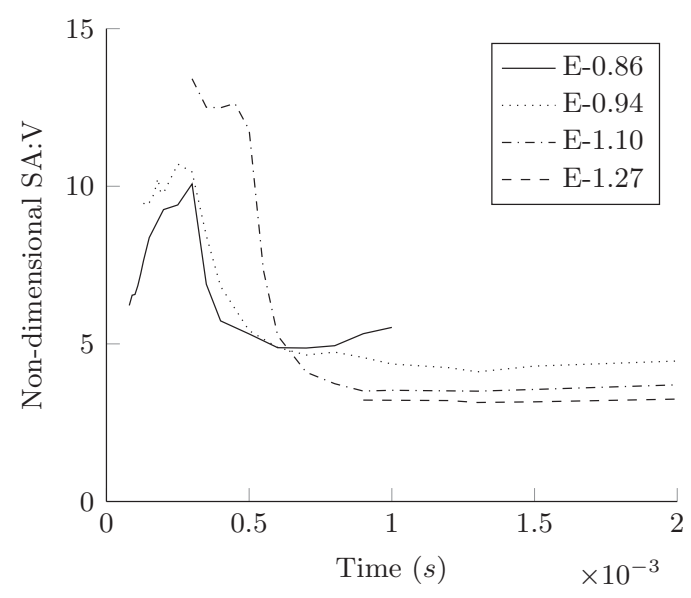

FIG. 9. Experimentally measured nondimensional surface-area-to-volume ratio. Measurements are taken from a height $0.7 \mathrm{~mm}$ above the free surface, omitting the base of the jet, and thus overestimate the surfacearea-to-volume ratio. Uncertainties are not shown in view of plot clarity.

compared to the volume of the spike jet. Disregarding the initial transients, the jet volume is shown to also be growing linearly with time.

Based on the quantities of generated free surface area and liquid volume we may draw interesting conclusions regarding the evolution of the surface jet. Figure 8(c) plots the nondimensional (based on the maximum bubble radius) surface-area-to-volume ratio of the free surface jet, $\mathrm{SA}: V^{*}=$ SA $R_{\max } / V$. This quantity describes the compactness of the jet and can be useful in deriving source terms for models such as the $\Sigma-\Upsilon$ model [48], where these variables are solved for and are therefore known. Interestingly, following the first few oscillation cycles, the surface-area-to-volume ratio of the jet is constant. This follows directly from the linear evolution of the surface area and volume shown in Figs. 8(a) and 8(b), but their ratio gives an estimation of the magnitude of the bubble-induced free surface stretching.

Data from the experiments may be used to support the above observation. The experimentally measured nondimensional surface-area-to-volume ratio, plotted in Fig. 9, remains constant after the first few cycles. A mismatch with the numerically predicted values is noticeable; the experimental data overestimate the surface-area-to-volume ratio and may be used only as qualitative indications. This is because the measurements are taken from a height $0.7 \mathrm{~mm}$ above the free surface and do not include the base of the jet, where the area-to-volume ratio is small.

Atomization of the free surface is realized at stages later than those discussed up to this point. However, a crucial factor to determine whether this will occur is the evolution of the free surface at the early stages. It would therefore be interesting to determine whether the interaction between the bubble and the free surface is able to produce sufficient stretching to allow for mechanisms such as the Rayleigh-Plateau instability to take over and atomize the interface at a later stage.

Based on the experimental study, we may estimate a critical (maximum) stand-off parameter for which atomization occurs. Figure 10 shows shadowgraph images depicting the very-late-time evolution of the free surface for the case where $\gamma=1.27$. This may be considered as a limit case for bubble-induced atomization, as the drop forms very close to the free surface and has no momentum left to travel away from it. Furthermore, in experiments where larger $\gamma$ values were considered, no droplets were observed detaching from the surface.

We attempt to make a link between early-stage interaction and bubble-induced free surface atomization using the jet compactness measure that was presented in Fig. 8(c). We postulate that the onset of atomization is related to a critical value of the nondimensional surface-area-to-volume 


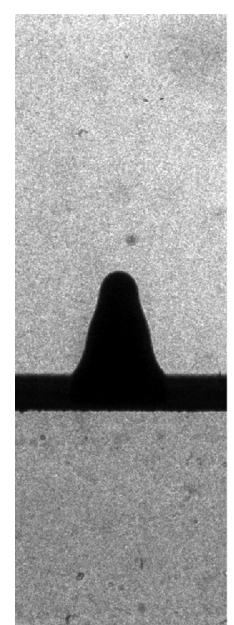

$t=3.0 \mathrm{~ms}$

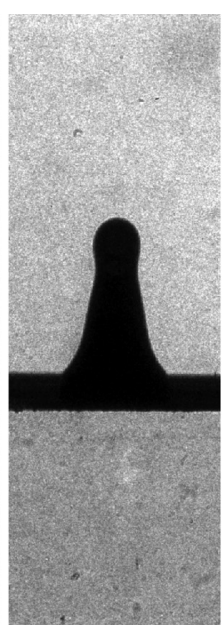

$t=5.0 \mathrm{~ms}$

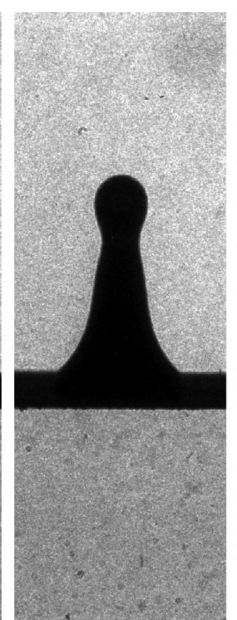

$t=7.0 \mathrm{~ms}$

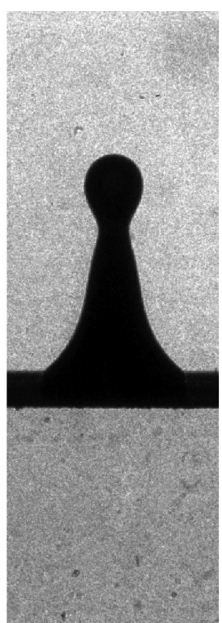

$t=9.0 \mathrm{~ms}$

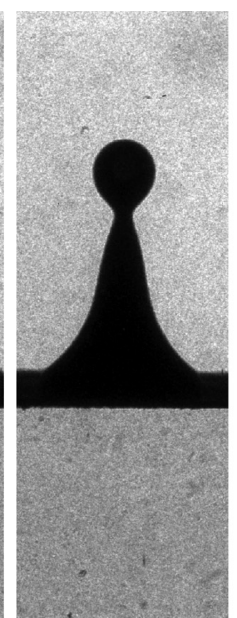

$t=11.0 \mathrm{~ms}$

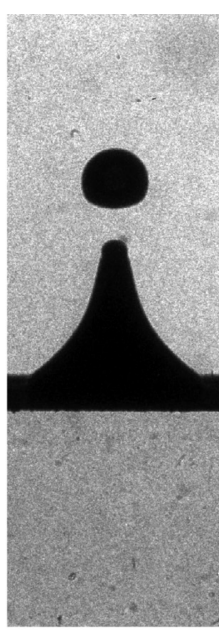

$t=13.0 \mathrm{~ms}$

FIG. 10. Late-time behavior of the bubble-free-surface system, case E-1.27. Depicted window size $6 \mathrm{~mm} \times$ $17 \mathrm{~mm}$.

ratio, $\left(\mathrm{SA}: V^{*}\right)_{\text {crit }}=\alpha$ (where $\alpha$ is a constant). Guided by the experimentally extracted limit case $(\gamma=1.27)$, and by fitting the asymptotic values of the data from the numerical simulations [Fig. 8(c)], we estimate the critical $\alpha$ value to be close to unity. However, as the simulations and experiments are not strictly equivalent (e.g. due to the idealized conditions in the simulations), we approximate this by assuming that the surface area of the jet (multiplied by the maximum bubble radius) needs to be larger than its volume in order for atomization to occur (SA: $V^{*} \geqslant 1$, i.e., $\alpha=1$ ). Second, an upper limit for the critical value $\alpha$ (effectively describing an uncertainty region) may be identified by observing that droplet formation has already commenced, within the simulated times (see Fig. 6), for cases where $\gamma \leqslant 0.92$, i.e., $\left(\text { SA: } V^{*}\right)_{\text {crit }} \simeq 1.98$ [Fig. 8(c)]. Figure 11 plots the asymptotic values of the nondimensional surface-area-to-volume ratio for different values

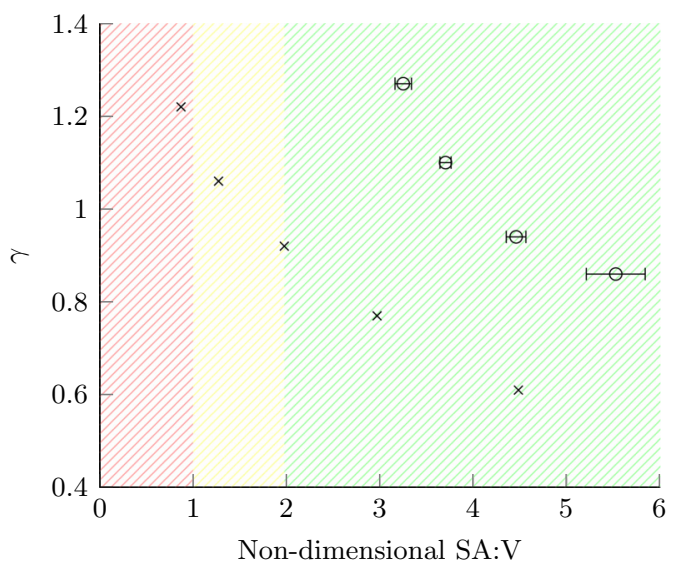

FIG. 11. Asymptotic behavior of nondimensional surface-area-to-volume ratio for different values of the nondimensional stand-off parameter $\gamma .(\times)$ Numerical simulations, $(\circ)$ experiments. The bars denote experimental uncertainty. The critical value $\alpha$ lies within the yellow region. The regions in green and red indicate where atomization is expected or not, respectively. 
of the nondimensional stand-off parameter $\gamma$, along with the regions emanating from the above rough approach at identifying the critical value $\alpha$ (depicted in different colors). The experimentally measured values of nondimensional surface-area-to-volume ratio are also included, although for the reason mentioned earlier, they may not be used to draw quantitative conclusions.

Of course, bubble-induced atomization is not a scale-free phenomenon, since surface tension and gravity can have a significant part in the evolution of the flow [28,29]. This means that the critical $\gamma$ values are not universal, but have a dependency on the system's size. It would be interesting to investigate whether the critical surface-area-to-volume ratio $\alpha$ can be used to identify the critical $\gamma$ values for atomization in systems of different sizes.

\section{CONCLUSIONS}

This work reported on an experimental and numerical investigation of the interaction between a cavitation bubble and an initially flat free surface. The behavior of the system was studied for a number of dimensionless distances that result in different interaction patterns and dynamics. Particular focus was given to the dynamics and deformation of the free surface.

Results from a series of simulations were used to characterize the process of bubble-induced atomization, by tracking the evolution of the jet volume and the generated liquid surface area. It was found that surface area is most rapidly generated during the second bubble cycle, and that at later times, the free surface evolves with a constant surface-area-to-volume ratio.

The latter quantity may be used to characterize the bubble-induced atomization process and has the potential of being used to improve the models that are employed when this process is part of a bigger picture (e.g., liquid fuel injection systems). Finally, a phenomenological criterion for determining whether the interaction between the bubble and the free surface is strong enough to allow for secondary mechanisms to atomize the free surface was proposed.

In the future, simulations will be carried out for bubbles of different sizes in an attempt to characterize the patterns, dynamics, and atomization characteristics of the system over a wide range of scales. Subsequent investigations could also involve the study of systems consisting of several bubbles and/or curved free surfaces.

\section{ACKNOWLEDGMENTS}

This work was carried out in the framework of the HAoS project, which has received funding from the European Union Horizon 2020 Research and Innovation program, Grant Agreement No. 675676. N.B. and Y.V. would like to express their gratitude to Professors J. Glimm and X. Li of Stony Brook University for the use of their front tracking framework.

[1] A. J. Coleman, J. E. Saunders, L. A. Crum, and M. Dyson, Acoustic cavitation generated by an extracorporeal shockwave lithotripter, Ultrasound Med. Biol. 13, 69 (1987).

[2] M. R. Bailey, V. A. Khokhlova, O. A. Sapozhnikov, S. G. Kargl, and L. A. Crum, Physical mechanisms of the therapeutic effect of ultrasound (a review), Acoust. Phys. 49, 369 (2003).

[3] A. Philipp and W. Lauterborn, Cavitation erosion by single laser-produced bubbles, J. Fluid Mech. 361, 75 (1998).

[4] K. S. Suslick, Sonochemistry, Science 247, 1439 (1990).

[5] A. H. Woodcock, C. F. Kientzler, A. B. Arons, and D. C. Blanchard, Giant condensation nuclei from bursting bubbles, Nature (London) 172, 1144 (1953).

[6] F. MacIntyre, Flow patterns in breaking bubbles, J. Geophys. Res. 77, 5211 (1972).

[7] J. Wu, Evidence of sea spray produced by bursting bubbles, Science 212, 324 (1981).

[8] H. Lhuissier and E. Villermaux, Bursting bubble aerosols, J. Fluid Mech. 696, 5 (2012). 
[9] C. F. Brasz, C. T. Bartlett, P. L. L. Walls, E. G. Flynn, Y. E. Yu, and J. C. Bird, Minimum size for the top jet drop from a bursting bubble, Phys. Rev. Fluids 3, 074001 (2018).

[10] J. C. W. Rogers and W. G. Szymczak, Computations of violent surface motions: Comparisons with theory and experiment, Philos. Trans. R. Soc. Lond. A 355, 649 (1997).

[11] E. Klaseboer, B. C. Khoo, and K. C. Hung, Dynamics of an oscillating bubble near a floating structure, J. Fluids Struct. 21, 395 (2005).

[12] J. Li and J.-L. Rong, Bubble and free surface dynamics in shallow underwater explosion, Ocean Eng. 38, 1861 (2011).

[13] H. P. Le, Progress and trends in ink-jet printing technology, J. Imaging Sci. Technol. 42, 49 (1998).

[14] C. B. Arnold, P. Serra, and A. Piqué, Laser direct-write techniques for printing of complex materials, MRS Bull. 32, 23 (2007).

[15] A. Dadvand, B. C. Khoo, and M. T. Shervani-Tabar, A collapsing bubble-induced microinjector: An experimental study, Exp. Fluids 46, 419 (2009).

[16] N. Kyriazis, P. Koukouvinis, and M. Gavaises, Numerical investigations on bubble-induced jetting and shock wave focusing: Application on a needle-free injection, Proc. R. Soc. A 475, 20180548 (2019).

[17] A. Sou, S. Hosokawa, and A. Tomiyama, Effects of cavitation in a nozzle on liquid jet atomization, Int. J. Heat Mass Transf. 50, 3575 (2007).

[18] H. K. Suh and C. S. Lee, Effect of cavitation in nozzle orifice on the diesel fuel atomization characteristics, Int. J. Heat Fluid Flow 29, 1001 (2008).

[19] F. Örley, T. Trummler, S. Hickel, M. S. Mihatsch, S. J. Schmidt, and N. A. Adams, Large-eddy simulation of cavitating nozzle flow and primary jet break-up, Phys. Fluids 27, 086101 (2015).

[20] M.-G. Mithun, P. Koukouvinis, and M. Gavaises, Numerical simulation of cavitation and atomization using a fully compressible three-phase model, Phys. Rev. Fluids 3, 064304 (2018).

[21] J. Zhou and M. Andersson, An analysis of surface breakup induced by laser-generated cavitation bubbles in a turbulent liquid jet, Exp. Fluids 61, 242 (2020).

[22] G. L. Chahine, Interaction between an oscillating bubble and a free surface, J. Fluids Eng. 99, 709 (1977).

[23] J. R. Blake and D. C. Gibson, Growth and collapse of a vapour cavity near a free surface, J. Fluid Mech. 111, 123 (1981).

[24] A. Patrascioiu, J. M. Fernández-Pradas, A. Palla-Papavlu, J. L. Morenza, and P. Serra, Laser-generated liquid microjets: Correlation between bubble dynamics and liquid ejection, Microfluid. Nanofluid. 16, 55 (2014).

[25] S. Zhang, S. P. Wang, and A. M. Zhang, Experimental study on the interaction between bubble and free surface using a high-voltage spark generator, Phys. Fluids 28, 032109 (2016).

[26] C. Ji, B. Li, J. Zou, and H. Yang, Interaction of two spark generated bubbles beneath free surface, Exp. Therm. Fluid Sci. 81, 76 (2017).

[27] Y. J. Kang and Y. Cho, Gravity-capillary jet-like surface waves generated by an underwater bubble, J. Fluid Mech. 866, 841 (2019).

[28] J. R. Blake, B. B. Taib, and G. Doherty, Transient cavities near boundaries Part 2. Free surface, J. Fluid Mech. 181, 197 (1987).

[29] Q. X. Wang, K. S. Yeo, B. C. Khoo, and K. Y. Lam, Nonlinear interaction between gas bubble and free surface, Comput. Fluids 25, 607 (1996).

[30] P. B. Robinson, J. R. Blake, T. Kodama, A. Shima, and Y. Tomita, Interaction of cavitation bubbles with a free surface, J. Appl. Phys. 89, 8225 (2001).

[31] A. Pearson, E. Cox, J. R. Blake, and S. R. Otto, Bubble interactions near a free surface, Eng. Anal. Bound. Elem. 28, 295 (2004).

[32] S. J. Lind and T. N. Phillips, The effect of viscoelasticity on the dynamics of gas bubbles near free surfaces, Phys. Fluids 25, 022104 (2013).

[33] P. Koukouvinis, M. Gavaises, O. Supponen, and M. Farhat, Simulation of bubble expansion and collapse in the vicinity of a free surface, Phys. Fluids 28, 052103 (2016).

[34] T. Li, A.-M. Zhang, S.-P. Wang, S. Li, and W.-T. Liu, Bubble interactions and bursting behaviors near a free surface, Phys. Fluids 31, 042104 (2019). 
[35] N. A. Hawker and Y. Ventikos, Interaction of a strong shockwave with a gas bubble in a liquid medium: A numerical study, J. Fluid Mech. 701, 59 (2012).

[36] http://www.ams.sunysb.edu/ linli/FronTier++_Manual/index.html.

[37] J. Glimm, E. Isaacson, D. Marchesin, and O. McBryan, Front tracking for hyperbolic systems, Adv. Appl. Math. 2, 91 (1981).

[38] J. Glimm and O. A. Mcbryan, A computational model for interfaces, Adv. Appl. Math. 6, 422 (1985).

[39] J. Glimm, J. W. Grove, X. L. Li, and D. C. Tan, Robust computational algorithms for dynamic interface tracking in three dimensions, SIAM J. Sci. Comput. 21, 2240 (2000).

[40] W. Bo, X. Liu, J. Glimm, and X. Li, A robust front tracking method: Verification and application to simulation of the primary breakup of a liquid jet, SIAM J. Sci. Comput. 33, 1505 (2011).

[41] N. Bempedelis and Y. Ventikos, A simplified approach for simulations of multidimensional compressible multicomponent flows: The grid-aligned ghost fluid method, J. Comput. Phys. 405, 109129 (2020).

[42] N. Bempedelis and Y. Ventikos, A simple ghost fluid method for compressible multicomponent flows with capillary effects, J. Comput. Phys. 424, 109861 (2021).

[43] N. Bempedelis and Y. Ventikos, Energy focusing in shock-collapsed bubble arrays, J. Fluid Mech. 900, A44 (2020).

[44] D. Obreschkow, M. Tinguely, N. Dorsaz, P. Kobel, A. De Bosset, and M. Farhat, The quest for the most spherical bubble: Experimental setup and data overview, Exp. Fluids 54, 1503 (2013).

[45] O. Supponen, P. Kobel, D. Obreschkow, and M. Farhat, The inner world of a collapsing bubble, Phys. Fluids 27, 091113 (2015).

[46] O. Supponen, D. Obreschkow, P. Kobel, and M. Farhat, Detailed jet dynamics in a collapsing bubble, J. Phys.: Conf. Ser. 656, 012038 (2015).

[47] M. Tinguely, D. Obreschkow, P. Kobel, N. Dorsaz, A. de Bosset, and M. Farhat, Energy partition at the collapse of spherical cavitation bubbles, Phys. Rev. E 86, 046315 (2012).

[48] A. Valet, A. A. Burluka, and R. Borghi, Development of a Eulerian model for the "atomization" of a liquid jet, Atomization Sprays 11, 619 (2001). 